\title{
Generic Submanifolds of Nearly Kaehler Manifolds with Certain Parallel Canonical Structure
}

\author{
Qingqing Zhu and Biaogui Yang \\ School of Mathematics and Computer Science, Fujian Normal University, Fuzhou 350108, China \\ Correspondence should be addressed to Biaogui Yang; bgyang@163.com
}

Received 30 June 2014; Accepted 17 August 2014; Published 29 October 2014

Academic Editor: Francesco Tornabene

Copyright (C) 2014 Q. Zhu and B. Yang. This is an open access article distributed under the Creative Commons Attribution License, which permits unrestricted use, distribution, and reproduction in any medium, provided the original work is properly cited.

The class of generic submanifold includes all real hypersurfaces, complex submanifolds, totally real submanifolds, and CRsubmanifolds. In this paper we initiate the study of generic submanifolds in a nearly Kaehler manifold from differential geometric point of view. Some fundamental results in this paper will be obtained.

\section{Introduction}

Nearly Kaehler manifolds have been studied intensively in the 1970's by Gray [1]. These nearly Kaehler manifolds are almost Hermitian manifolds with almost complex structure $J$ for which the tensor field $\bar{\nabla} J$ is skew-symmetric. In particular, the complex structure is nonintegrable if the manifold is non-Kaehler. As we all know, there are two natual types of submanifolds of nearly Kaehler (or more generally, almost Hermitian) manifold, namely, almost complex and totally real submanifolds. Almost complex submanifolds are submanifolds whose tangent spaces are invariant under $J$ and totally real submanifolds are opposite. A well known example is the nearly Kaehler 6-dimensional sphere which has been studied by many authors (see, e.g., [2-7]).

In 1981, Chen introduced preliminary the differential geometry of real submanifolds in a Kaehler manifold ([8]) and gave some basic formulas and definitions. Inspired by that paper, we will generalize some important formulas and proprerties in a Kaehler manifold to a nearly Kaehler manifold. The paper is organized as follows: the basic on nearly Kaehler manifolds and submanifold theory will be recapitulated in Section 2. In Section 3, we give the integrability conditions of the two natural distributions $\mathscr{H}$ and $\mathscr{H}^{\perp}$ associated with a generic submanifold of nearly Kaehler manifold. Finally, we consider generic submanifolds with one of its canonical structures to be parallel. These results enable us to prove the following theorem.
Theorem 1. Let $M$ be a generic submanifold in a nearly Kaehler manifold $\bar{M}$. If $P$ (or $F$ ) is parallel, then the holomorphic distribution $\mathscr{H}$ is intergrable.

The operator $P$ (or $F$ ) is a canonical structure as the following paper introduced.

\section{Preliminaries}

An almost Hermitian manifold $(\bar{M}, g, J)$ is a manifold endowed with an almost complex structure $J$, that is, compatible with the metric $g$, that is, an endomorphism $J: T \bar{M} \rightarrow$ $T \bar{M}$ such that $J_{P}^{2}=-I d$ for every $p \in \bar{M}$ and $g(J X, J Y)=$ $g(X, Y)$. A nearly Kaehler manifold is an almost Hermitian manifold with the extra condition that the $(1,2)$-tensor field $G=\bar{\nabla} J$ is skew-symmetric:

$$
\left(\bar{\nabla}_{X} J\right) Y+\left(\bar{\nabla}_{Y} J\right) X=0
$$

for every $X, Y \in T \bar{M}$. Here $\bar{\nabla}$ stands for the Levi-Civita connection of the metric $g$. The tensor field $G$ on $\bar{M}$ satisfies the following properties $([1,2])$ :

$$
G(X, Y)=-G(Y, X)
$$




$$
\begin{aligned}
G(X, J Y) & =-J G(X, Y), \\
g(G(X, Y), Z) & =-g(G(X, Z), Y), \\
g(G(X, Y), Z) & =g(G(Y, Z), X)=g(G(Z, X), Y),
\end{aligned}
$$

where $X, Y$, and $Z$ are arbitrary vector fields on $\bar{M}$.

We denote the metrics of $\bar{M}$ and its submanifold $M$ by the same letter $g, T M$ is the tangent bundle of $M$, and $T^{\perp} M$ is the normal bundle of $M$. If $\nabla$ and $\nabla^{\perp}$ denote the Riemannian connection induced on $M$ and the connection in the normal bundle $T^{\perp} M$, respectively, then the Gauss and Weingarten formulas are

$$
\begin{gathered}
\bar{\nabla}_{X} Y=\nabla_{X} Y+\sigma(X, Y), \\
\bar{\nabla}_{X} \xi=-A_{\xi} X+\nabla_{X}^{\perp} \xi,
\end{gathered}
$$

where $X, Y \in T M$ and $\xi \in T^{\perp} M$. The second fundamental form $\sigma$ and the shape operator $A_{\xi}$ are related to each other by

$$
g(\sigma(X, Y), \xi)=g\left(A_{\xi} X, Y\right) .
$$

For any vector field $X$ tangent to $M$, we put

$$
J X=P X+F X,
$$

where $P X$ and $F X$ are the tangential and normal components of $J X$, respectively. Then $P$ is an endomorphism of the tangent bundle TM and $F$ is a normal-bundle-valued 1-form on TM. For any vector field $\xi$ normal to $M$, we put

$$
J \xi=t \xi+f \xi
$$

where $t \xi$ and $f \xi$ are the tangential and normal components of $J \xi$, respectively. Then $f \xi$ is an endomorphism of the normal bundle $T^{\perp} M$ and $t$ is a tangent-bundle-valued 1-form on $T^{\perp} M$.

For a submanifold $M$ in a nearly Kaehler manifold $\bar{M}$ we define

$$
\mathscr{H}_{x}=T_{x} M \cap J T_{x} M,
$$

the holomorphic tangent space of $M$ at $x . \mathscr{H}_{x}$ is the maximal complex subspace of $T_{x} \bar{M}$ which is contained in $T_{x} M$.

Similar to [8], we will give several definitions as follows.

Definition 2. A submanifold $M$ in a nearly Kahler manifold (or in an almost complex manifold in general) is called a generic submanifold if $\operatorname{dim} \mathscr{H}_{x}$ is constant along $M$ and $\mathscr{H}_{x}$ defines a differentiable distribution on $M$, called the holomorphic distribution.

Definition 3. A generic submanifold $M$ in a nearly Kaehler manifold is a totally real (resp., complex) submanifold if $J T M \subseteq T^{\perp} M$ (resp., JTM = TM).

For a generic submanifold $M$ in a nearly Kaehler manifold $\bar{M}$, the orthogonal complementary distribution $\mathscr{H}^{\perp}$, called the purely real distribution, satisfies

$$
\begin{gathered}
\mathscr{H}_{x} \perp \mathscr{H}_{x}^{\perp}, \quad P \mathscr{H}_{x}^{\perp} \subseteq \mathscr{H}_{x}^{\perp}, \\
\mathscr{H}_{x}^{\perp} \cap J \mathscr{H}_{x}^{\perp}=\{0\} .
\end{gathered}
$$

From (9) it is clear that the normal-bundle-valued 1-form $F$ induces an isomorphism from $\mathscr{H}_{x}^{\perp}$ onto $F \mathscr{H}_{x}^{\perp}$. Let $v_{x}$ be the vector space of holomorphic normal vectors to $M$ at $x$, or simply the holomorphic normal space of $M$ at $x$; that is,

$$
v_{x}=T_{x}^{\perp} M \cap J T_{x}^{\perp} M .
$$

Then $v_{x}$ defines a differentiable vector subbundle of $T^{\perp} M$. we have that

$$
\begin{gathered}
T^{\perp} M=F \mathscr{H}^{\perp} \oplus v, \quad t\left(T^{\perp} M\right)=\mathscr{H}^{\perp}, \\
g\left(F \mathscr{H}^{\perp}, v\right)=0 .
\end{gathered}
$$

\section{Integrability}

In this section we study the integrability of the holomorphic distribution $\mathscr{H}$ and the purely real distribution $\mathscr{H}^{\perp}$. First we give the following.

Lemma 4. Let $M$ be a generic submanifold in a nearly Kaehler manifold $\bar{M}$. Then

$$
g(\sigma(X, J Y)-\sigma(J X, Y), \eta)=g\left(2 G(X, Y)^{\perp}, \eta\right),
$$

for any vector $X, Y \in \mathscr{H}$ and $\eta \in v$.

Proof. From (2) and (6), we obtain

$$
\begin{aligned}
g(J \sigma(X, U), \eta) & =g\left(J \bar{\nabla}_{U} X, \eta\right) \\
& =g\left(\bar{\nabla}_{U} J X-G(U, X)^{\perp}, \eta\right) \\
& =g\left(\sigma(J X, U)+G(X, U)^{\perp}, \eta\right),
\end{aligned}
$$

where $X \in \mathscr{H}, U \in T M$, and $\eta \in v$. This implies that

$$
g(J \sigma(X, Y), \eta)=g\left(\sigma(J X, Y)+G(X, Y)^{\perp}, \eta\right),
$$

where $X, Y \in \mathscr{H}$ and $\eta \in v$. Since the second fundamental form $\sigma$ is symmetric, we have

$$
g\left(\sigma(J X, Y)+G(X, Y)^{\perp}, \eta\right)=g\left(\sigma(J Y, X)+G(Y, X)^{\perp}, \eta\right) .
$$

From the equations above, we prove the lemma.

Proposition 5. Let $M$ be a generic submanifold in a nearly Kaehler manifold $\bar{M}$. Then the holomorphic distribution $\mathscr{H}$ is integrable if and only if

$$
g(\sigma(X, J Y)-\sigma(J X, Y), F Z)=g\left(2 G(X, Y)^{\perp}, F Z\right),
$$

for any vector fields $X, Y \in \mathscr{H}$, and $Z \in \mathscr{H}^{\perp}$.

Proof. Since $\bar{M}$ is nearly Kaehlerian, using formulas (2) and (6), we have

$$
\begin{aligned}
& \sigma(X, J Y)-\sigma(J X, Y) \\
&=\bar{\nabla}_{X} J Y-\nabla_{X} J Y-\bar{\nabla}_{Y} J X+\nabla_{Y} J X \\
&= G(X, Y)+J \bar{\nabla}_{X} Y-G(Y, X) \\
& \quad-J \bar{\nabla}_{Y} X+\nabla_{Y} J X-\nabla_{X} J Y \\
&= 2 G(X, Y)+J[X, Y]+\nabla_{Y} J X-\nabla_{X} J Y .
\end{aligned}
$$


So we get

$$
\begin{gathered}
\sigma(X, J Y)-\sigma(J X, Y)-2 G(X, Y) \\
=J[X, Y]+\nabla_{Y} J X-\nabla_{X} J Y
\end{gathered}
$$

for any vector field $X, Y$ in $\mathscr{H}$. If the holomorphic distribution $\mathscr{H}$ is integrable, the right-hand-side of (22) lies in TM; thus we obtain $\sigma(X, J Y)-\sigma(J X, Y)=2 G(X, Y)^{\perp}$. In particular, we have (20). Conversely, if (20) holds, then by Lemma 4 and (14) we have $\sigma(X, J Y)-\sigma(J X, Y)=2 G(X, Y)^{\perp}$ for any vectors $X, Y$ in $\mathscr{H}$. Thus by (22) we obtain $J[X, Y]=\nabla_{X} J Y-\nabla_{Y} J X$. Since $\nabla_{X} J Y-\nabla_{Y} J X$ is tangent to $M$, this implies that $[X, Y]$ lies in $\mathscr{H}$. Thus we proved the proposition from the Frobenius theorem.

Proposition 6. Let $M$ be a generic submanifold in a nearly kaehler manifold $M$. Then the purely real distribution $\mathscr{H}^{\perp}$ is integrable if and only if

$$
P\left\{A_{F W} Z-A_{F Z} W+\nabla_{W} P Z-\nabla_{Z} P W\right\}-2 J G(W, Z)
$$

lies in $\mathscr{H}^{\perp}$, for any vector fields $Z, W \in \mathscr{H}^{\perp}$.

Proof. For any vector fields $Z, W \in \mathscr{H}^{\perp},(6)$ gives

$$
J\left(\bar{\nabla}_{Z} W\right)=J \nabla_{Z} W+J \sigma(Z, W),
$$

and it follows immediately from (6), (7), and (9) that

$$
\begin{aligned}
J\left(\bar{\nabla}_{Z} W\right)= & \bar{\nabla}_{Z} J W-G(Z, W) \\
= & \bar{\nabla}_{Z} P W+\bar{\nabla}_{Z} F W-G(Z, W) \\
= & \nabla_{Z} P W+\sigma(Z, P W)-A_{F W} Z \\
& +D_{Z} F W-G(Z, W) .
\end{aligned}
$$

From which we obtain

$$
\begin{gathered}
-\nabla_{Z} W-\sigma(Z, W)=J \nabla_{Z} P W+J \sigma(Z, P W) \\
-J A_{F W} Z+J D_{Z} F W-J G(Z, W) .
\end{gathered}
$$

Comparing the tangential parts, we have

$$
\begin{aligned}
\nabla_{Z} W= & -P \nabla_{Z} P W-t \sigma(Z, P W) \\
& +P A_{F W} Z-t D_{Z} F W-G(Z, J W)^{\top}
\end{aligned}
$$

Thus we get

$$
\begin{aligned}
{[Z, W]=} & P\left\{A_{F W} Z-A_{F Z} W+\nabla_{W} P Z-\nabla_{Z} P W\right\} \\
& +t\left\{\sigma(W, P Z)-\sigma(Z, P W)+D_{W} F Z-D_{Z} F W\right\} \\
& -2 J G(W, Z)
\end{aligned}
$$

Since $t\left(T^{\perp} M\right)=\mathscr{H}^{\perp}$, this implies that $[Z, W]$ lies in $\mathscr{H}^{\perp}$ if and only if (23) lies in $\mathscr{H}^{\perp}$. The proposition is proved.
Theorem 7. Let $M$ be a generic submanifold in a nearly Kaehler manifold $\bar{M}$. If $\mathscr{H}$ is integrable and its leaves are totally geodesic in $M$, then

$$
g\left((G+J \sigma)(\mathscr{H}, \mathscr{H}), \mathscr{H}^{\perp}\right)=0 .
$$

Proof. Since $\mathscr{H}$ is integrable and its leaves are totally geodesic in $M$, we have $\nabla_{X} Y \in \mathscr{H}$, for any $X, Y \in \mathscr{H}$. So $g\left(\nabla_{X} Z, Y\right)=$ $g\left(\nabla_{X} Y, Z\right)=0$, and we can get $\nabla_{X} Z \in \mathscr{H}^{\perp}$ for any vector $X \in \mathscr{H}$ and $Z \in \mathscr{H}^{\perp}$. From (2), (6), (7), and (9), we get

$$
\begin{aligned}
g & \left(\nabla_{X} Z, J Y\right) \\
& =-g\left(J \bar{\nabla}_{X} Z, Y\right) \\
& =g\left(G(X, Z)-\bar{\nabla}_{X} J Z, Y\right) \\
& =g(G(X, Z), Y)-g\left(\bar{\nabla}_{X} P Z, Y\right)-g\left(\bar{\nabla}_{X} F Z, Y\right) \\
& =g(G(X, Z), Y)-g\left(\nabla_{X} P Z, Y\right)+g\left(A_{F Z} X, Y\right),
\end{aligned}
$$

which implies that

$$
\begin{aligned}
-g & (G(X, Y), Z)+g(\sigma(X, Y), J Z) \\
& =-g(G(X, Y)+J \sigma(X, Y), Z)=0 .
\end{aligned}
$$

This proves the theorem.

Theorem 8. Let $M$ be a generic submanifold in a nearly Kaehler manifold $\bar{M}$. If $\mathscr{H}^{\perp}$ is integrable and its leaves are totally geodesic in $M$, then

$$
g\left((G-J \sigma)\left(\mathscr{H}, \mathscr{H}^{\perp}\right), \mathscr{H}^{\perp}\right)=0 .
$$

Proof. Under the hypothesis, for any vector fields $X$ in $\mathscr{H}$ and $Z, W$ in $\mathscr{H}^{\perp}$, it follows from (3), (6), (7), and (9) that

$$
\begin{aligned}
g\left(\nabla_{Z} X, W\right)= & g\left(J \bar{\nabla}_{Z} X, J W\right) \\
= & g\left(-G(Z, X)+\bar{\nabla}_{Z} J X, P W+F W\right) \\
= & g(G(X, Z), J W)+g\left(\bar{\nabla}_{Z} J X, P W\right) \\
& +g\left(\bar{\nabla}_{Z} J X, F W\right) \\
= & g(G(X, Z), J W)+g(\sigma(J X, Z), F W) \\
= & -g(J G(X, Z)+J \sigma(J X, Z), W) \\
= & g(G(J X, Z)-J \sigma(J X, Z), W) .
\end{aligned}
$$

That is,

$$
g(G(J X, Z)-J \sigma(J X, Z), W)=0 .
$$

From this we obtain the theorem.

\section{Generic Submanifolds with Parallel Canonical Structure}

For the endomorphism $P: T M \rightarrow T M$, we put

$$
\left(\bar{\nabla}_{X} P\right) Y=\nabla_{X} P Y-P \nabla_{X} Y,
$$


for any vector fields $X, Y \in T M$. The endomorphism $P$ is said to be parallel if $\bar{\nabla}_{X} P=0$ for any vector $X \in T M$. From (6), (7), and (9) we can obtain the following:

$$
\begin{aligned}
J \nabla_{X} Y+J \sigma(X, Y)= & J \bar{\nabla}_{X} Y \\
= & \bar{\nabla}_{X} J Y-G(X, Y) \\
= & \bar{\nabla}_{X} P Y+\bar{\nabla}_{X} F Y-G(X, Y) \\
= & \nabla_{X} P Y+\sigma(X, P Y) \\
& -A_{F Y} X+D_{X} F Y-G(X, Y) .
\end{aligned}
$$

That is,

$$
\begin{aligned}
& P \nabla_{X} Y+F \nabla_{X} Y+t \sigma(X, Y)+f \sigma(X, Y) \\
& \quad=\nabla_{X} P Y+\sigma(X, P Y)-A_{F Y} X+D_{X} F Y-G(X, Y) .
\end{aligned}
$$

By comparing the tagential parts, we have the following:

$$
\left(\bar{\nabla}_{X} P\right) Y=t \sigma(X, Y)+A_{F Y} X+G(X, Y)^{\top} .
$$

Therefore, for any vector fields $X, Y, Z \in T M$, we have

$$
\begin{aligned}
g\left(\left(\bar{\nabla}_{X} P\right) Y, Z\right)= & g\left(t \sigma(X, Y)+A_{F Y} X+G(X, Y)^{\top}, Z\right) \\
= & -g(\sigma(X, Y), J Z)+g\left(A_{F Y} X, Z\right) \\
& +g\left(G(X, Y)^{\top}, Z\right) \\
= & g\left(A_{F Y} Z-A_{F Z} Y+G(Y, Z)^{\top}, X\right) .
\end{aligned}
$$

So, we obtain the Lemma as follows.

Lemma 9. Let $M$ be a generic submanifold in a nearly Kaehler manifold $\bar{M}$. The $P$ is parallel, that is, $\bar{\nabla} P=0$, if and only if

$$
G(U, V)^{\top}=A_{F V} U-A_{F U} V,
$$

for any vectors $U, V \in T M$.

Theorem 10. Let $M$ be a generic submanifold in a nearly Kaehler manifold $\bar{M}$. If $P$ is parallel, then

(i) $G(U, X)^{\top}=-A_{F U} X$, for any vector fields $X \in \mathscr{H}$ and $U \in T M$

(ii) the holomorphic distribution $\mathscr{H}$ is intergrable.

Proof. From Lemma 9, for any vector fields $X \in \mathscr{H}$ and $U \in$ $T M$, we know $F X=0$; this implies that $G(U, X)^{\top}=-A_{F U} X$. On the other hand, for any vector fields $X, Y \in \mathscr{H}, G(X, Y)^{\top}=$ $-A_{F X} Y=0$, then $G(X, Y)$ is normal to $M$. By (i), we can get

$$
g(G(U, X), Y)=g\left(-A_{F U} X, Y\right)
$$

that is,

$$
g(G(X, Y)-J \sigma(X, Y), U)=0
$$

for any vector fields $X, Y \in \mathscr{H}$ and $U \in T M$. The equations above imply that

$$
g(\sigma(X, Y), F Z)=0,
$$

for any vector fields $X, Y \in \mathscr{H}$ and $Z \in \mathscr{H}^{\perp}$. These give

$$
g(\sigma(X, J Y)-\sigma(J X, Y), F Z)=g\left(2 G(X, Y)^{\perp}, F Z\right) .
$$

From Proposition 5, the theorem holds.

For the normal bundle-valued 1-form $F$, we put

$$
\left(\bar{\nabla}_{X} F\right) Y=\nabla_{X} F Y-F \nabla_{X} Y \text {. }
$$

For any vector fields $X, Y \in T M$. The endomorphism $F$ is said to be parallel if $\bar{\nabla}_{X} F=0$ for any vector $X \in T M$. By comparing the normal parts of (37), we have the following:

$$
\left(\bar{\nabla}_{X} F\right) Y=f \sigma(X, Y)-\sigma(X, P Y)+G(X, Y)^{\perp},
$$

for any vectors $X, Y \in T M$. Hence, for any vector field $\xi \in$ $T^{\perp} M$, it follows from (4), (8), and (10) that

$$
\begin{aligned}
g & \left(\left(\bar{\nabla}_{X} F\right) Y, \xi\right) \\
& =g\left(f \sigma(X, Y)-\sigma(X, P Y)+G(X, Y)^{\perp}, \xi\right) \\
& =g(J \sigma(X, Y), \xi)-g(\sigma(X, P Y), \xi)+g(G(X, Y), \xi) \\
& =-g(\sigma(X, Y), f \xi)-g(\sigma(X, P Y), \xi)+g(G(X, Y), \xi) \\
& =-g\left(A_{f \xi} Y+A_{\xi} P Y-G(Y, \xi)^{\top}, X\right) .
\end{aligned}
$$

From which we obtain the Lemma as follows.

Lemma 11. Let $M$ be a generic submanifold in a nearly Kaehler manifold $\bar{M}$. The $F$ is parallel, that is, $\bar{\nabla} F=0$, if and only if

$$
A_{f \xi} X+A_{\xi} P X=G(X, \xi)^{\top},
$$

for any vectors $X \in T M$ and $\xi \in T^{\perp} M$.

Theorem 12. Let $M$ be a generic submanifold in a nearly Kaehler manifold $\bar{M}$. If $F$ is parallel, then the holomorphic distribution $\mathscr{H}$ is intergrable.

Proof. From (46) we have,

$$
\begin{aligned}
g( & \left.\left(\bar{\nabla}_{X} F\right) Y, \xi\right) \\
= & g\left(f \sigma(X, Y)-\sigma(X, P Y)+G(X, Y)^{\perp}, \xi\right) \\
= & g(J \sigma(X, Y), \xi)-g(\sigma(X, P Y), \xi) \\
& +g\left(G(X, Y)^{\perp}, \xi\right),
\end{aligned}
$$

for any vectors $X, Y \in \mathscr{H}$ and $\xi \in T^{\perp} M$. Since $F$ is parallel, then

$$
g(J \sigma(X, Y), \xi)-g(\sigma(X, P Y), \xi)+g\left(G(X, Y)^{\perp}, \xi\right)=0
$$


That is,

$$
J \sigma(X, Y)=\sigma(X, J Y)-G(X, Y)^{\perp}
$$

This implies that

$$
g(\sigma(X, J Y)-\sigma(J X, Y), F Z)=g\left(2 G(X, Y)^{\perp}, F Z\right),
$$

for any vectors $X, Y \in \mathscr{H}$ and $Z \in \mathscr{H}^{\perp}$. From Proposition 5, the theorem holds.

\section{Conflict of Interests}

The authors declare that there is no conflict of interests regarding the publication of this paper.

\section{Acknowledgments}

This work was supported by the Grant no. 2011J05001 of NSF of Fujian Province, China, and the Grant no. JA11052 of the Fund of the Education Department of Fujian Province, China, and was partially supported by Grant no. 11171139 of NSFC.

\section{References}

[1] A. Gray, "Nearly Kähler manifold," Journal of Differential Geometry, vol. 4, pp. 283-309, 1970.

[2] F. Belgun and A. Moroianu, "Nearly Kähler 6-manifolds with reduced holonomy," Annals of Global Analysis and Geometry, vol. 19, no. 4, pp. 307-319, 2001.

[3] B. Chen and T. Nagano, "Totally geodesic submanifolds of symmetric spaces, I," Duke Mathematical Journal, vol. 44, no. 4, pp. 745-755, 1977.

[4] F. Dillen, L. Verstraelen, and L. Vrancken, "Almost complex submanifolds of a 6-dimensional sphere II," Kodai Mathematical Journal, vol. 10, pp. 161-171, 1987.

[5] N. Ejiri, "Totally real submanifolds in a 6-sphere," Proceedings of the American Mathematical Society, vol. 83, no. 4, pp. 759-763, 1981.

[6] A. Gray, "Minimal varieties and almost Hermitian submanifolds," Michigan Mathematical Journal, vol. 12, pp. 273-287, 1965.

[7] A. Gray, "Almost complex submanifolds of the six sphere," Proceedings of the American Mathematical Society, vol. 20, pp. 277-279, 1969.

[8] B.-Y. Chen, "Differential geometry of real submanifolds in a Kähler manifold," Monatshefte für Mathematik, vol. 91, no. 4, pp. 257-274, 1981. 


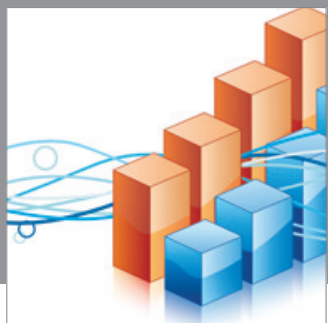

Advances in

Operations Research

mansans

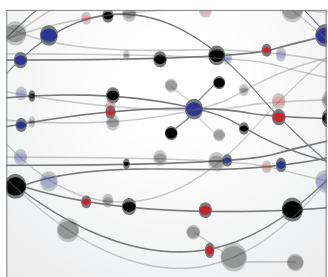

The Scientific World Journal
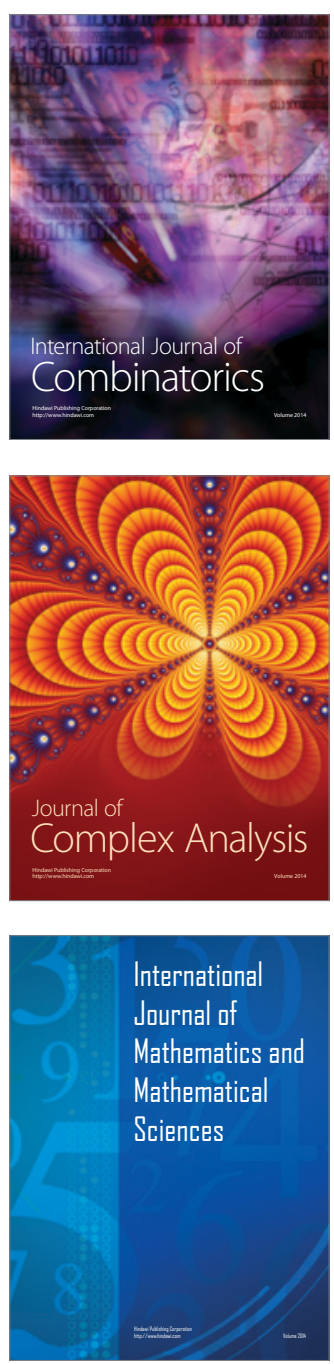
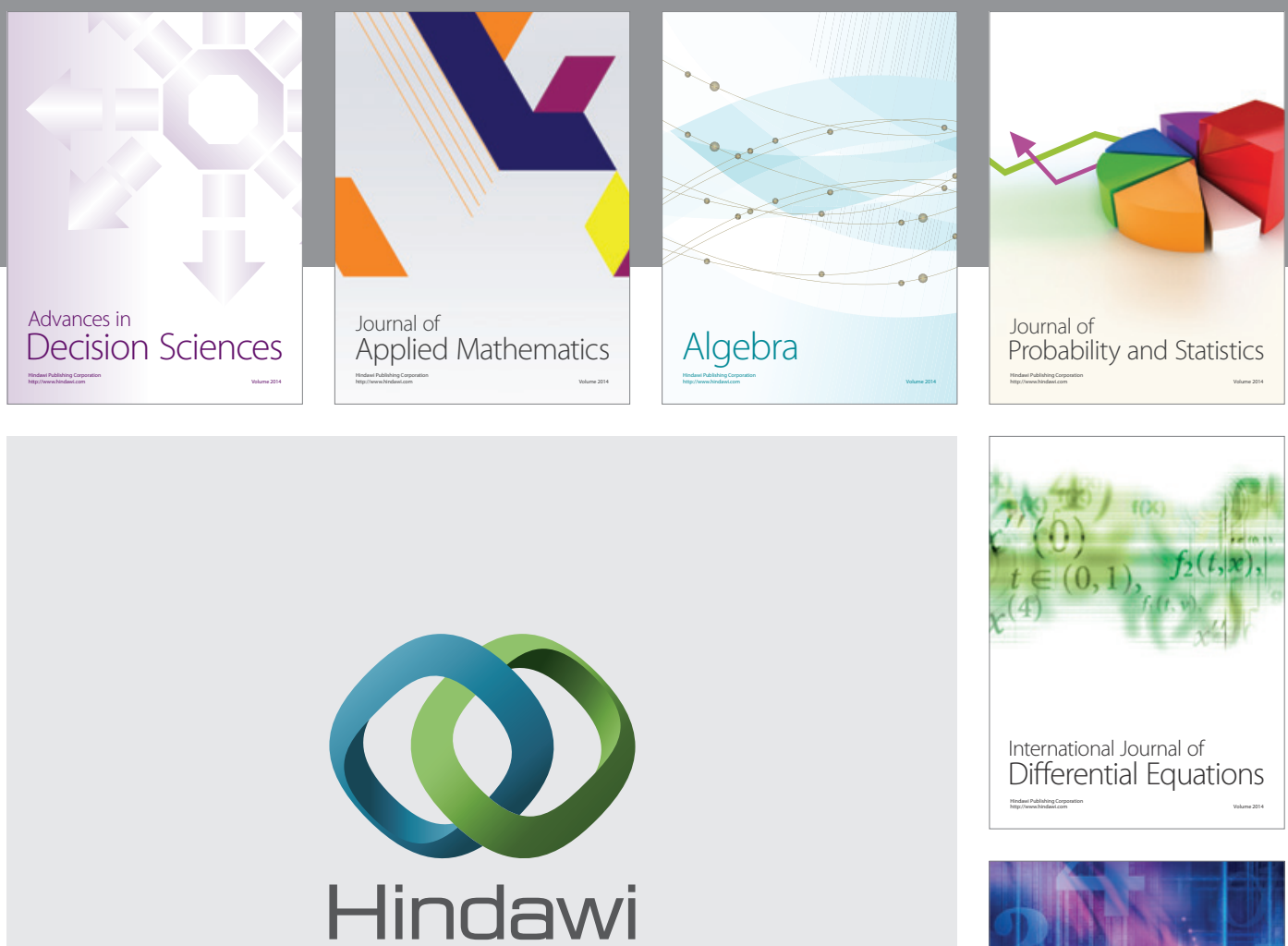

Submit your manuscripts at http://www.hindawi.com
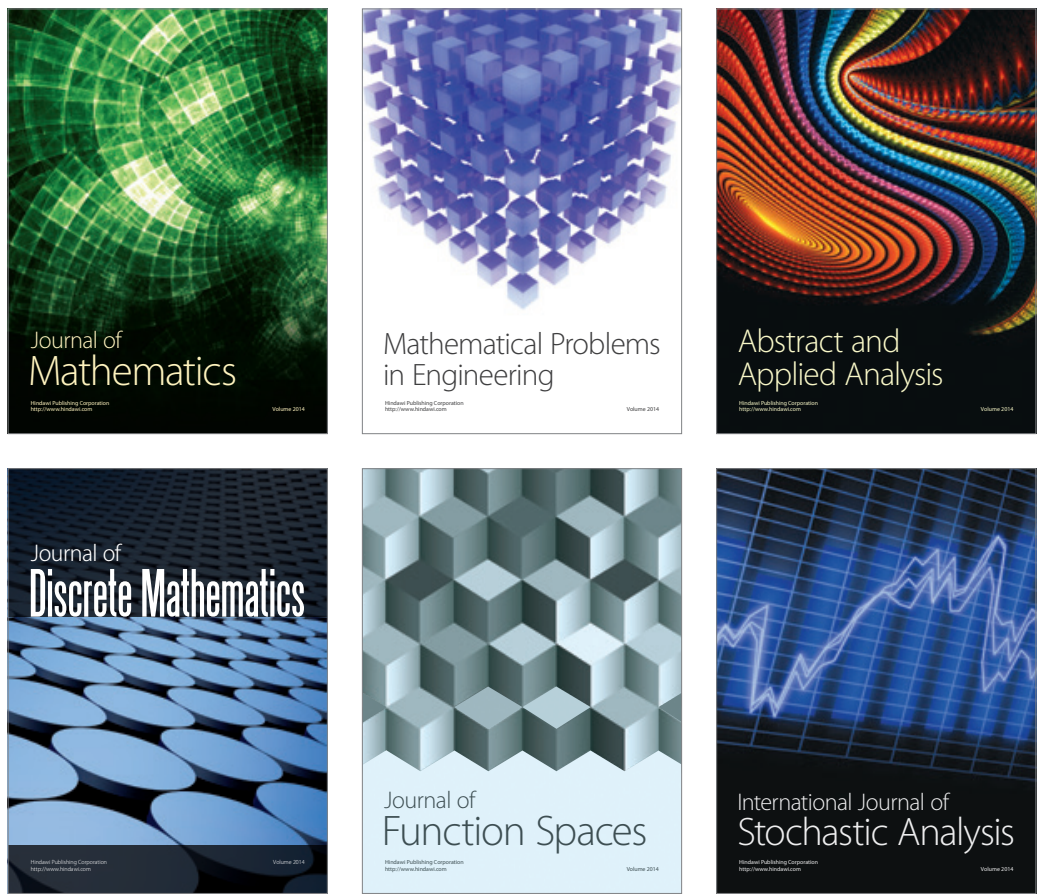

Journal of

Function Spaces

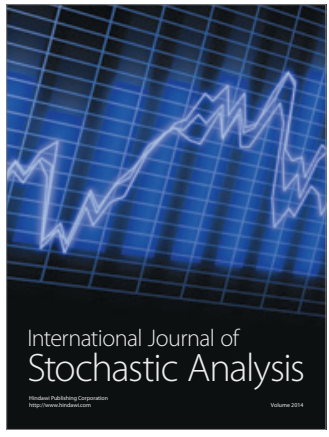

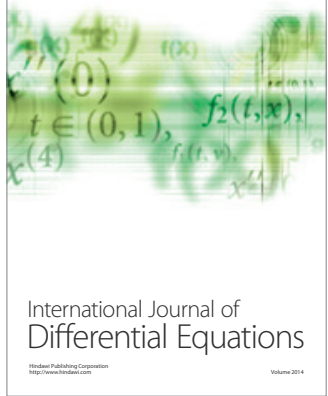
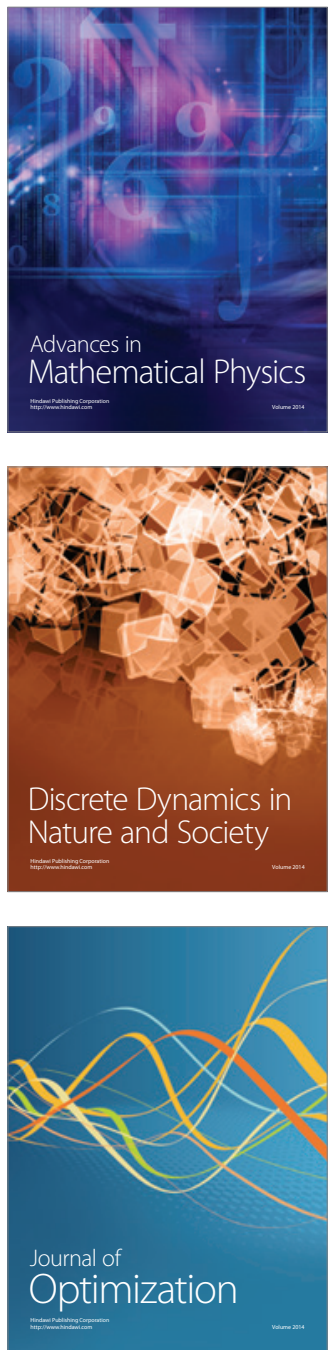